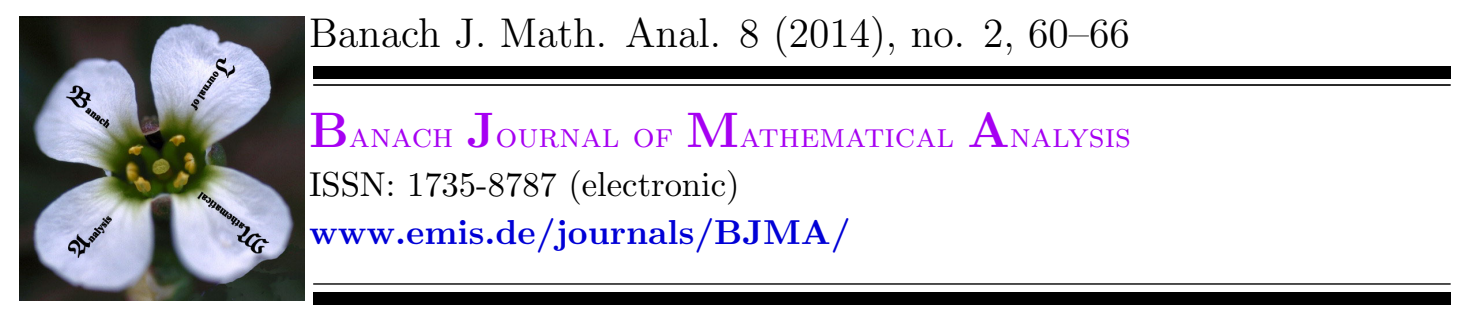

\title{
PSEUDOQUOTIENTS ON COMMUTATIVE BANACH ALGEBRAS
}

\author{
DRAGU ATANASIU ${ }^{1}$, PIOTR MIKUSIŃSKI ${ }^{2 *}$ AND ANGELA SIPLE ${ }^{2}$ \\ Communicated by M. Abel
}

\begin{abstract}
We consider pseudoquotient extensions of positive linear functionals on a commutative Banach algebra $\mathcal{A}$ and give conditions under which the constructed space of pseudoquotients can be identified with all Radon measures on the structure space $\hat{\mathcal{A}}$.
\end{abstract}

\section{INTRODUCTION}

Let $\mathcal{A}$ be a Banach algebra with involution. The structure space of $\mathcal{A}$, denoted by $\hat{\mathcal{A}}$, is the set of all multiplicative linear functionals on $\mathcal{A}$. We use $\hat{x}$ to denote the Gelfand transform of $x$, that is $\hat{x}(\xi)=\xi(x)$ for any $x \in \mathcal{A}$ and $\xi \in \hat{\mathcal{A}}$.

A linear functional $f: \mathcal{A} \rightarrow \mathbb{C}$ is called positive, if

$$
f\left(x^{*} x\right) \geq 0 \text {, for all } x \in \mathcal{A} .
$$

The set of all positive linear functionals on an algebra $\mathcal{A}$ is denoted by $\mathcal{P}(\mathcal{A})$. The following theorem (attributed to Maltese in [5]) describes $\mathcal{P}(\mathcal{A})$ in terms of measures on $\hat{\mathcal{A}}$.

Theorem 1.1. Let $\mathcal{A}$ be a commutative Banach algebra with a bounded approximate identity and an isometric and symmetric involution. Let $f$ be a linear

Date: Received: Aug. 6, 2013; Accepted: Sep. 8, 2013.

* Corresponding author.

2010 Mathematics Subject Classification. Primary 46J05; Secondary 43A32, 43A35, 28C05.

Key words and phrases. Banach algebra, structure space, positive linear functional, pseudoquotient, Radon measure. 
functional on $\mathcal{A}$. Then $f \in \mathcal{P}(\mathcal{A})$ if and only if

$$
f(x)=\int_{\hat{\mathcal{A}}} \hat{x}(\xi) d \mu_{f}(\xi),
$$

for all $x \in \mathcal{A}$, with respect to a unique positive Radon measure on $\hat{\mathcal{A}}$ of total variation $\|f\|$.

By a Radon measure on $\hat{\mathcal{A}}$ we mean a continuous linear functional on the space $\mathcal{K}(\hat{\mathcal{A}})$ of continuous complex-valued functions on $\hat{\mathcal{A}}$ with compact support equipped with the standard inductive limit topology. The space of all such measures will be denoted by $\mathcal{M}(\hat{\mathcal{A}})$. The set of all positive Radon measures on $\hat{\mathcal{A}}$ will be denoted by $\mathcal{M}_{+}(\hat{\mathcal{A}})$ and the set of all bounded positive Radon measures on $\hat{\mathcal{A}}$ will be denoted by $\mathcal{M}_{+}^{b}(\hat{\mathcal{A}})$. The topology of $\mathcal{M}_{+}^{b}(\hat{\mathcal{A}})$ is the topology of uniform convergence on $\hat{\mathcal{A}}$ and the topology of $\mathcal{M}_{+}(\hat{\mathcal{A}})$ is the topology of uniform convergence on compact subsets of $\hat{\mathcal{A}}$.

Let $\mathcal{F}: \mathcal{P}(\mathcal{A}) \rightarrow \mathcal{M}_{+}^{b}(\hat{\mathcal{A}})$ be the map defined by Maltese's theorem, that is, $\mathcal{F}(f)=\mu_{f}$. In terms of the introduced notation, Theorem 1.1 states that $\mathcal{F}$ is an isometry between $\mathcal{P}(\mathcal{A})$ and $\mathcal{M}_{+}^{b}(\hat{\mathcal{A}})$. In this paper we give conditions under which $\mathcal{P}(\mathcal{A})$ can be extended to a space of pseudoquotients $\mathcal{B}(\mathcal{P}(\mathcal{A}), \mathcal{S})$ such that $\mathcal{F}$ can be extended to a bijection between $\mathcal{B}(\mathcal{P}(\mathcal{A}), \mathcal{S})$ and $\mathcal{M}_{+}(\hat{\mathcal{A}})$.

In the next section we recall the construction of pseudoquotients and its basic properties. The construction of pseudoqutients was introduced in [8] under the name of "generalized quotients." The motivation for the idea, early developments, and later modifications, are discussed in [9]. The construction of pseudoquotients has desirable properties. For instance, it preserves the algebraic structure of $X$ and has good topological properties. There is growing evidence that pseudoquotients can be a useful tool (see, for example, [1], [2], or [3]).

In Section 3 we formulate and prove the main result of the paper. In the final section we discuss some examples. We also show that the result in [2] is a special case of the construction presented here.

\section{Pseudoquotients}

Let $X$ be a nonempty set and let $S$ be a commutative semigroup acting on $X$ injectively. The relation

$$
(x, \varphi) \sim(y, \psi) \quad \text { if } \quad \psi x=\varphi y
$$

is an equivalence in $X \times S$. We define $\mathcal{B}(X, S)=(X \times S) / \sim$. Elements of $\mathcal{B}(X, S)$ are called pseudoquotients. The equivalence class of $(x, \varphi)$ will be denoted by $\frac{x}{\varphi}$. Thus $\frac{x}{\varphi}=\frac{y}{\psi}$ means $\psi x=\varphi y$.

Elements of $X$ can be identified with elements of $\mathcal{B}(X, S)$ via the embedding $\iota: X \rightarrow \mathcal{B}(X, S)$ defined by $\iota(x)=\frac{\varphi x}{\varphi}$, where $\varphi$ is an arbitrary element of $S$. The action of $S$ can be extended to $\mathcal{B}(X, S)$ via $\varphi \frac{x}{\psi}=\frac{\varphi x}{\psi}$. If $\varphi \frac{x}{\psi}=\iota(y)$, for some $y \in X$, we simply write $\varphi \frac{x}{\psi} \in X$ and $\varphi \frac{x}{\psi}=y$. For instance, we have $\varphi \frac{x}{\varphi}=x$.

In the case $X$ is a topological space or a convergence space and $S$ is a commutative semigroup of continuous injections acting on $X$, then we can define a 
convergence in $\mathcal{B}(X, S)$ as follows: If, for a sequence $F_{n} \in \mathcal{B}(X, S)$, there exist a $\varphi \in S$ and an $F \in \mathcal{B}(X, S)$ such that $\varphi F_{n}, \varphi F \in X$, for all $n \in \mathbb{N}$, and $\varphi F_{n} \rightarrow \varphi F$ in $X$, then we write $F_{n} \stackrel{I}{\rightarrow} F$ in $\mathcal{B}(X, S)$. In other words, $F_{n} \stackrel{I}{\rightarrow} F$ in $\mathcal{B}(X, S)$ if $F_{n}=\frac{x_{n}}{\varphi}, F=\frac{x}{\varphi}$, and $x_{n} \rightarrow x$ in $X$, for some $x_{n}, x \in X$ and $\varphi \in S$.

This convergence is sometimes referred to as type $I$ convergence. It is quite natural, but it need not be topological. For this reason we prefer to use the convergence defined as follows: $F_{n} \rightarrow F$ in $\mathcal{B}(X, S)$ if every subsequence $\left(F_{p_{n}}\right)$ of $\left(F_{n}\right)$ has a subsequence $\left(F_{q_{n}}\right)$ such that $F_{q_{n}} \stackrel{I}{\rightarrow} F$.

It is easy to show that the embedding $\iota: X \rightarrow \mathcal{B}(X, S)$, as well as the extension of any $\varphi \in S$ to a map $\varphi: \mathcal{B}(X, S) \rightarrow \mathcal{B}(X, S)$ defined above, are continuous.

\section{THE MAIN RESULT}

In this section we will assume $\mathcal{A}$ to be a nonunital commutative Banach algebra with bounded approximate identities and an isometric and symmetric involution. In addition, we assume that $\mathcal{A}$ satisfies the following condition:

$\boldsymbol{\Sigma}$ There exists a sequence $a_{1}, a_{2}, \ldots \in \mathcal{A}$ such that $\hat{a}_{1}, \hat{a}_{2}, \ldots \in \mathcal{K}(\hat{\mathcal{A}})$ and for every $\xi \in \hat{\mathcal{A}}$ there is an $n$ such that $\hat{a}_{n}(\xi) \neq 0$.

For $a \in \mathcal{A}$, by $\Lambda_{a}$ we denote the operation on linear functionals on $\mathcal{A}$ defined by $\left(\Lambda_{a} f\right)(x)=f(a x)$. Let

$$
\mathcal{S}=\left\{\Lambda_{a}: \hat{a}>0 \text { on } \hat{\mathcal{A}}\right\}
$$

Lemma 3.1. If $\mathcal{A}$ satisfies $\boldsymbol{\Sigma}$, then $\mathcal{S}$ is a nonempty commutative semigroup of injective maps acting on $\mathcal{P}(\mathcal{A})$.

Proof. Without loss of generality, we may assume that $\hat{a}_{n} \geq 0$ and that for every $\xi \in \hat{\mathcal{A}}$ there exists an $n$ such that $\hat{a}_{n}(\xi)>0$ (otherwise, we take $a_{n} a_{n}^{*}$ instead of $\left.a_{n}\right)$. If we choose $\lambda_{n}>0$ such that $\sum_{n=1}^{\infty}\left\|\lambda_{n} a_{n}\right\|<\infty$ and define $a=\sum_{n=1}^{\infty} \lambda_{n} a_{n}$, then $\Lambda_{a} \in \mathcal{S}$.

Clearly, $\mathcal{S}$ is a commutative semigroup. Let $f \in \mathcal{P}(\mathcal{A})$ and $\Lambda_{a} \in \mathcal{S}$. By Maltese's theorem [5], $f(x)=\int_{\hat{\mathcal{A}}} \hat{x}(\xi) d \mu(\xi)$ for some $\mu \in \mathcal{M}_{+}^{b}(\hat{\mathcal{A}})$. Thus

$$
\left(\Lambda_{a} f\right)(x)=f(a x)=\int_{\hat{\mathcal{A}}} \widehat{a x}(\xi) d \mu(\xi)=\int_{\hat{\mathcal{A}}} \hat{x}(\xi) \hat{a}(\xi) d \mu(\xi) .
$$

Since $\hat{a}(\xi)>0$ for all $\xi \in \hat{\mathcal{A}}$, $\hat{a}$ is a positive bounded function on $\hat{\mathcal{A}}$. Thus $\tilde{\mu}=\hat{a} \mu \in \mathcal{M}_{+}^{b}(\hat{\mathcal{A}})$ and $\Lambda_{a} f(x)=\int_{\hat{\mathcal{A}}} \hat{x}(\xi) d \tilde{\mu}(\xi)$. Consequently $\Lambda_{a} f \in \mathcal{P}(\mathcal{A})$.

If $\Lambda_{a} f=0$, then

$$
0=f(a x)=\int_{\hat{\mathcal{A}}} \widehat{a x}(\xi) d \mu(\xi)=\int_{\hat{\mathcal{A}}} \hat{x}(\xi) \hat{a}(\xi) d \mu(\xi),
$$

for all $x$ in $\mathcal{A}$. Therefore $\hat{a} \mu=0$ and $\mu=0$, because $\hat{a}>0$. Thus

$$
f(x)=\int_{\hat{\mathcal{A}}} \hat{x}(\xi) d \mu(\xi)=0 .
$$

Hence $\Lambda_{a}$ is injective. 
The map $\mathcal{F}: \mathcal{P}(\mathcal{A}) \rightarrow \mathcal{M}_{+}^{b}(\hat{\mathcal{A}})$ defined by Maltese's theorem, can be extended to a map $\mathcal{F}: \mathcal{B}(\mathcal{P}(\mathcal{A}), \mathcal{S}) \rightarrow \mathcal{M}_{+}(\hat{\mathcal{A}})$ in the natural way:

$$
\mathcal{F}\left(\frac{f}{\Lambda_{a}}\right)=\frac{\mathcal{F}(f)}{\hat{a}}=\frac{1}{\hat{a}} \mu_{f} .
$$

It is clear that $\mathcal{F}$ is well-defined and that it is injective.

Theorem 3.2. Let $\mathcal{A}$ be a nonunital commutative Banach algebra with a bounded approximate identity and an isometric and symmetric involution. If $\mathcal{A}$ satisfies $\boldsymbol{\Sigma}$, then the extended $\mathcal{F}$ defined by (3.1) is an bijection from $\mathcal{B}(\mathcal{P}(\mathcal{A}), \mathcal{S})$ to $\mathcal{M}_{+}(\hat{\mathcal{A}})$.

Proof. It suffices to show that $\mathcal{F}$ is surjective. Let $\mu \in \mathcal{M}_{+}(\hat{\mathcal{A}})$. There are $a_{n} \in \mathcal{A}$ such that $\hat{a}_{n} \geq 0$, supp $\hat{a}_{n}$ is compact, and such that for every $\xi \in \hat{\mathcal{A}}$ there exists an $n$ such that $\hat{a}_{n}(\xi)>0$. Then $\hat{a}_{n} \mu$ is a finite positive Radon measure on $\hat{\mathcal{A}}$ for every $n \in \mathbb{N}$. There exist positive numbers $\lambda_{1}, \lambda_{2}, \ldots$ such that $\sum_{n=1}^{\infty} \lambda_{n} \hat{a}_{n} \mu$ defines a finite positive Radon measure on $\hat{\mathcal{A}}$. By Maltese's theorem there exist $f \in \mathcal{P}(\mathcal{A})$ such that

$$
\mu_{f}=\sum_{n=1}^{\infty} \lambda_{n} \hat{a}_{n} \mu .
$$

Without loss of generality, we can assume that the numbers $\lambda_{1}, \lambda_{2}, \ldots$ are chosen such that

$$
\sum_{n=1}^{\infty}\left\|\lambda_{n} a_{n}\right\|<\infty .
$$

Let $a=\sum_{n=1}^{\infty} \lambda_{n} a_{n}$. Then $\Lambda_{a} \in \mathcal{S}$ and $\sum_{n=1}^{\infty} \lambda_{n} \hat{a}_{n} \mu=\hat{a} \mu$. Thus

$$
\mathcal{F}\left(\frac{f}{\Lambda_{a}}\right)=\frac{\mu_{f}}{\hat{a}}=\frac{\hat{a} \mu}{\hat{a}}=\mu .
$$

Theorem 3.3. The map $\mathcal{F}: \mathcal{B}(\mathcal{P}(\mathcal{A}), \mathcal{S}) \rightarrow \mathcal{M}_{+}(\hat{\mathcal{A}})$ is a sequential homeomorphism.

Proof. If $F_{n} \stackrel{I}{\rightarrow} F$ in $\mathcal{B}(\mathcal{P}(\mathcal{A}), \mathcal{S})$, then $F_{n}=\frac{f_{n}}{\Lambda_{a}}, F=\frac{f}{\Lambda_{a}}$, and $f_{n} \rightarrow f$ in $\mathcal{P}(\mathcal{A})$ for some $f_{n}, f \in \mathcal{P}(\mathcal{A})$, where $f_{n} \rightarrow f$ means $f_{n}(x) \rightarrow f(x)$ for all $x \in \mathcal{A}$. Consequently,

$$
\int_{\hat{\mathcal{A}}} \hat{x}(\xi) d \mu_{f_{n}}(\xi) \rightarrow \int_{\hat{\mathcal{A}}} \hat{x}(\xi) d \mu_{f}(\xi)
$$

for all $x \in \mathcal{A}$. Since the involution in $\mathcal{A}$ is symmetric and $\Gamma(\mathcal{A})=\{\hat{x}: x \in \mathcal{A}\}$ strongly separates points in $\hat{\mathcal{A}}$ (see, for example, Theorem 2.2.7 in [6]), we obtain

$$
\int_{\hat{\mathcal{A}}} \varphi(\xi) d \mu_{f_{n}}(\xi) \rightarrow \int_{\hat{\mathcal{A}}} \varphi(\xi) d \mu_{f}(\xi)
$$

for all $\varphi \in \mathcal{K}(\hat{\mathcal{A}})$. Therefore,

$$
\int_{\hat{\mathcal{A}}} \varphi(\xi) \frac{d \mu_{f_{n}}(\xi)}{\hat{a}(\xi)} \rightarrow \int_{\hat{\mathcal{A}}} \varphi(\xi) \frac{d \mu_{f}(\xi)}{\hat{a}(\xi)}
$$


for all $\varphi \in \mathcal{K}(\hat{\mathcal{A}})$, which means that $\mathcal{F}\left(F_{n}\right) \rightarrow \mathcal{F}(F)$ in $\mathcal{M}_{+}(\hat{\mathcal{A}})$.

Now assume $\mu_{n}, \mu \in \mathcal{M}_{+}(\hat{\mathcal{A}})$ and

$$
\int_{\hat{\mathcal{A}}} \varphi(\xi) d \mu_{n}(\xi) \rightarrow \int_{\hat{\mathcal{A}}} \varphi(\xi) d \mu(\xi)
$$

for all $\varphi \in \mathcal{K}(\hat{\mathcal{A}})$. There exist $\lambda_{k}>0, k \in \mathbb{N}$, such that $\sum_{k=1}^{\infty} \lambda_{k} \hat{a}_{k} \mu_{n}$ is a finite measure for all $n \in \mathbb{N}$ and $\Lambda_{a} \in \mathcal{S}$, where $a=\sum_{k=1}^{\infty} \lambda_{k} a_{k}$. Let

$$
f_{n}=\mathcal{F}^{-1}\left(\sum_{k=1}^{\infty} \lambda_{k} \hat{a}_{k} \mu_{n}\right)=\mathcal{F}^{-1}\left(\hat{a} \mu_{n}\right)
$$

and

$$
f=\mathcal{F}^{-1}\left(\sum_{k=1}^{\infty} \lambda_{k} \hat{a}_{k} \mu\right)=\mathcal{F}^{-1}(\hat{a} \mu) .
$$

Then $\frac{f_{n}}{\Lambda_{a}}=\mathcal{F}^{-1}\left(\mu_{n}\right)$ and $\frac{f}{\Lambda_{a}}=\mathcal{F}^{-1}(\mu)$. Moreover,

$$
f_{n}(x)=\int_{\hat{\mathcal{A}}} \hat{x}(\xi) \hat{a}(\xi) d \mu_{n}(\xi) \rightarrow \int_{\hat{\mathcal{A}}} \hat{x}(\xi) \hat{a}(\xi) d \mu(\xi)=f(x)
$$

for every $x \in \mathcal{A}$. Therefore $\frac{f_{k}}{\Lambda_{a}} \stackrel{I}{\rightarrow} \frac{f}{\Lambda_{a}}$ in $\mathcal{B}(\mathcal{P}(\mathcal{A}), \mathcal{S})$.

\section{EXAMPLES}

In this section we give some examples of spaces where the assumptions of Theorem 3.2 are satisfied.

4.1. Normal algebras. Let $\mathcal{A}$ be a commutative Banach algebra. We say that $\mathcal{A}$ is normal [7], if for every compact $K \subset \hat{\mathcal{A}}$ and closed $E \subset \hat{\mathcal{A}}$ such that $K \cap E=\emptyset$, there exists $x \in \mathcal{A}$ such that

$$
\hat{x}(\xi)=1 \text { for } \xi \in K \text { and } \hat{x}(\xi)=0 \text { for } \xi \in E .
$$

If $\mathcal{A}$ is a normal commutative Banach algebra and $\hat{\mathcal{A}}$ is $\sigma$-compact, then $\mathcal{A}$ satisfies condition $\boldsymbol{\Sigma}$. Indeed, if $\hat{\mathcal{A}}$ is $\sigma$-compact, there are compact sets $K_{n} \subset \hat{\mathcal{A}}$ such that $\hat{\mathcal{A}}=\cup_{n=0}^{\infty} K_{n}$ and $K_{n} \subset K_{n+1}^{\circ}$ for all $n \in \mathbb{N}$, where $K_{n+1}^{\circ}$ is the interior of $K_{n+1}$. Since $\mathcal{A}$ is regular, for every $n \in \mathbb{N}$ there exists $b_{n} \in \mathcal{A}$ such that

$$
\hat{b}_{n}(\xi)=\left\{\begin{array}{ll}
1 & \text { if } \xi \in K_{n} \\
0 & \text { if } \xi \notin K_{n+1}^{\circ}
\end{array} .\right.
$$

Let $a_{n}=b_{n} b_{n}^{*}$. Then $\hat{a}_{n}=\left|\hat{b}_{n}\right|^{2} \geq 0$ and $K_{n} \subset \operatorname{supp} \hat{a}_{n} \subset K_{n+2}$. Clearly, for every $\xi \in \hat{\mathcal{A}}$, there exists $n$ such that $\hat{a}_{n}>0$.

Note that a regular commutative Banach algebra is normal, [7]. 
4.2. Algebras with $\sigma$-compact-open structure spaces. For our next example we use Shilov's idempotent theorem [10].

Theorem 4.1 (Shilov). Let $\mathcal{A}$ be a commutative Banach algebra. If $K$ is a compact and open subset of $\hat{\mathcal{A}}$, then there is a unique idempotent $a \in \mathcal{A}$ such that $\hat{a}$ is the characteristic function of $K$.

Let $\mathcal{A}$ be a commutative Banach algebra such that $\hat{\mathcal{A}}$ is $\sigma$-compact-open, that is, $\hat{\mathcal{A}}=\cup_{n=0}^{\infty} K_{n}$ where $K_{n}$ are disjoint compact and open sets in the Gelfand topology in $\hat{\mathcal{A}}$. Since, by Shilov's idempotent theorem, for every $n \in \mathbb{N}$ there exist a unique idempotent $a_{n} \in \mathcal{A}$ such that $\operatorname{supp} \hat{a}_{n}=K_{n}, \mathcal{A}$ satisfies condition $\Sigma$.

4.3. Locally compact groups. Let $G$ be a locally compact abelian group. A continuous function $f: G \rightarrow \mathbb{C}$ is called positive definite if

$$
\sum_{k, l=1}^{n} c_{k} \overline{c_{l}} f\left(x_{l}^{-1} x_{k}\right) \geq 0
$$

for all $c_{1}, \ldots, c_{n} \in \mathbb{C}$ and $x_{1}, \ldots, x_{n} \in G$ for any $n \in \mathbb{N}$. We denote the cone of positive definite functions on $G$ by $\mathcal{P}_{+}(G)$. A character $\alpha$ on $G$ is a continuous homomorphism from $G$ into the unit circle group $\mathbb{T}$. Let $\widehat{G}$ denote the group of characters. By Bochner's theorem [4], $f \in \mathcal{P}_{+}(G)$ if and only if there exists a unique bounded positive Radon measure $\mu_{f}$ on $\widehat{G}$ such that

$$
f(x)=\int_{\widehat{G}} \hat{x} d \mu_{f}
$$

In [2] it was shown that, if $\widehat{G}$ is $\sigma$-compact, then the map $f \mapsto \mu_{f}$ defined by Bochner's theorem can be extended to a map from a space of pseudoquotients to all positive measures on $\widehat{G}$. That space of pseudoquotients was $\mathcal{B}\left(\mathcal{P}_{+}(G), \mathcal{S}\right)$ where

$$
\mathcal{S}=\left\{\varphi \in L^{1}(G): \widehat{\varphi}(\xi)>0 \text { for all } \xi \in \widehat{G}\right\} .
$$

We will show that this extension is a special case of the extension presented in this note.

Since the convolution algebra $L^{1}(G)$ is regular, it satisfies $\boldsymbol{\Sigma}$, as indicated in 4.1. For $\alpha \in \widehat{G}$ we define $\varphi_{\alpha}: L^{1}(G) \rightarrow \mathbb{C}$ by

$$
\varphi_{\alpha}(f)=\int_{G} f(x) \overline{\alpha(x)} d x
$$

where $d x$ indicates the integral with respect to the Haar measure on $G$. The map $\alpha \mapsto \varphi_{\alpha}$ is a bijection from $\widehat{G}$ onto $\widehat{L^{1}(G)}$ (see, for example, [6]). This allows us to identify $\mathcal{M}_{+}(\widehat{G})$ and $\mathcal{M}_{+}\left(\widehat{L^{1}(G)}\right)$. If $f$ is a positive definite function on $G$, we define a positive functional on $L^{1}(G)$ by

$$
F(\varphi)=\int_{G} f(x) \varphi(x) d x
$$


and a map from $\mathcal{B}\left(\mathcal{P}_{+}(G), \mathcal{S}\right)$ to $\mathcal{B}\left(L^{1}(G), \mathcal{S}\right)$ by $\frac{f}{\varphi} \mapsto \frac{F}{\Lambda_{\tilde{\varphi}}}$, where $\tilde{\varphi}(x)=\varphi\left(x^{-1}\right)$.

Since $\mathcal{B}\left(L^{1}(G), \mathcal{S}\right)$ is isomorphic with $\mathcal{M}_{+}\left(\widehat{L^{1}(G)}\right)$, by Theorem 3.2, there is a bijection from $\mathcal{B}\left(\mathcal{P}_{+}(G), \mathcal{S}\right)$ to $\mathcal{M}_{+}(\hat{G})$.

\section{REFERENCES}

1. D. Atanasiu and P. Mikusiński, The Fourier transform of Levy measures on a semigroup, Integral Transform. Spec. Funct. 19 (2008), 537-543.

2. D. Atanasiu and P. Mikusiński, Fourier transform of Radon measures on locally compact groups, Integral Transform. Spec. Funct. 21 (2010), 815-821.

3. D. Atanasiu, P. Mikusiński, and D. Nemzer, An algebraic approach to tempered distributions, J. Math. Anal. Appl. 384 (2011), 307-319.

4. G.B. Folland, A Course in Abstract Harmonic Analysis, CRC Press, Boca Raton, 1995.

5. M. Fragoulopoulou, Topological Algebras with Involution, North-Holland Mathematics Studies 200, Elsevier Science B.V., Amsterdam, 2005.

6. E. Kaniuth, A Course in Commutative Banach Algebras, Springer, New York, 2008.

7. R. Larsen, Banach Algebras an Introduction, Marcel Dekker Inc., New York, 1973.

8. P. Mikusiński, Generalized quotients with applications in analysis, Methods Appl. Anal. 10 (2003), 377-386.

9. P. Mikusiński, Boehmians and pseudoquotients, Appl. Math. Inf. Sci. 5 (2011), 1-13.

10. T. W. Palmer, Banach algebras and the general theory of *algebras, Vol. I, Cambridge, New York, 1994.

${ }^{1}$ University of BorÅs, BorÅs, Sweden

E-mail address: Dragu.Atanasiu@hb.se

2 Department of Mathematics, University of Central Florida, Orlando, FL 32816, USA.

E-mail address: piotr.mikusinski@ucf.edu

E-mail address: asiple@knights.ucf.edu 